\section{Red neural por defecto y enfermedad de Alzheimer}

\author{
FERNANDO VERGARA E. ${ }^{1,2}$, MARÍA ISABEL BEHRENS ${ }^{1,3}$
}

\section{Default mode network and Alzheimer's disease}

Brain functioning is mainly intrinsic, not primarily reflexive. This is supported by the high energy requirements of the resting brain (20\% of all the energy consumed) which only marginally increases with changes in brain activity. Modern neuroimaging and neurophysiological techniques have led to the discovery of the so called brain default mode network (DMN), a constellation of brain regions which support brain activity at rest and whose discharges decrease during task-induced activities. Another characteristic of the DMN are the elevated levels of aerobic glycolysis (Warburg effect), that is, metabolism of glucose to lactic acid in the presence of sufficient levels of oxygen. In Alzheimer's disease there is amyloid deposition and metabolic disruption at the $D M N$ regions. Changes in connectivity among the different nodes of the DMN and its connections with the hippocampus have been reported. The characteristics of the $D M N$ and its relation to Alzheimer's disease are discussed. This issue is of interest in the pathogenesis and possibly for its usefulness as a biomarker of the disease.

(Rev Med Chile 2013; 141: 375-380).

Key words: Alzheimer disease; Dementia; Nerve net.

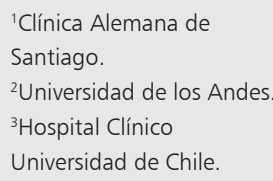

${ }^{1}$ Clínica Alemana de Santiago.

${ }^{2}$ Universidad de los Andes ${ }^{3}$ Hospital Clínico

Universidad de Chile.

Financiamiento: MIB: Fondecyt 1110189.

Recibido el 22 de mayo de 2012, aceptado el 15 de junio de 2012

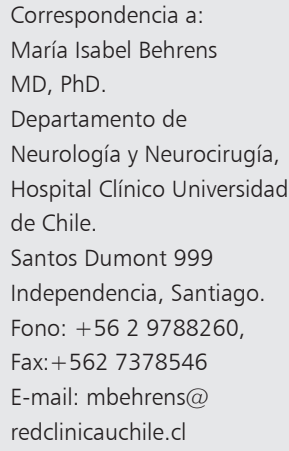

$\mathrm{D}$ urante el siglo XX se propusieron dos puntos de vista sobre el funcionamiento del cerebro. El primero podemos asimilarlo a la posición empiricista de John Locke, quien consideraba el cerebro una tabula rasa, en la cual la mente no tiene cualidades innatas y donde los conocimientos son fruto de la experiencia. Dentro de esta concepción, Sherrington propuso que el funcionamiento del sistema nervioso y la conducta dependen de reflejos complejos resultantes de las aferencias sensoriales. En una posición extrema, el cerebro permanecería inactivo hasta recibir impulsos sensoriales que son capaces de generar las respuestas apropiadas. Este modelo simple de aferencias/eferencias ha sido útil y generó el concepto de campo receptivo, donde la función de la neurona se define por las aferencias sensoriales que la llevan a un nivel de excitación máximo con la consecuente generación de potenciales de acción eferentes ${ }^{1}$. Esta postura ha sido la más utilizada en la investigación del sistema nervioso central incluyendo más recientemente las neuroimágenes funcionales. Es fácil trazar experimentos cuida- dosamente diseñados y controlados midiendo las respuestas cerebrales a diferentes estímulos.

El segundo punto de vista hace énfasis en un funcionamiento endógeno de los circuitos neurales. Brown, un discípulo de Sherrington, describió cómo la médula espinal de gatos decerebrados y deaferentados era capaz de generar conductas motoras de marcha, en ausencia de todo tipo de aferencias sensoriales ${ }^{2}$. Esto lo llevó a postular la presencia de circuitos funcionales autónomos o de un generador de patrones centrales (GPC), en el cual las aferencias sensitivas juegan un rol modulador. Dos GPC clásicos son los circuitos espinales de locomoción y los de tronco que regulan la respiración. Estos generadores centrales también pueden ser aplicados a circuitos neocorticales, que a pesar de ser modulados por aferencias sensitivas son capaces de generar respuestas en ausencia de ellas ${ }^{3}$.

La neurobiología actual es más acorde con el segundo punto de vista, con la posición de Immanuel Kant, en la cual la mente humana no es un mero receptor pasivo de impresiones sensoriales 
sino que más bien tiene esquemas a priori, prexistentes, tales como espacio, tiempo y causalidad. Uno de los exponentes más claros de esta posición es Rodolfo Llinás quien propone que la conciencia, igual que la locomoción representa más bien una actividad intrínseca del sistema nervioso, modulada, pero no generada por las aferencias sensoriales ${ }^{4}$. Recientemente, Dehaene y Brannon ${ }^{5}$ han planteado un programa de investigación, denominado Kantiano, que pretende entender cómo aparecen las intuiciones básicas, cómo éstas se relacionan con los mecanismos neurales, cuál de estos aspectos surgen independientemente de la experiencia y cuáles pueden ser enriquecidos por el entrenamiento y la educación.

Hacia fines del siglo XX se comenzó a plantear que el cerebro humano está organizado en una serie de redes neurales funcionales que interactúan en forma flexible para la realización de las diferentes funciones cognitivas. Las redes neurocognitivas contienen grupos de neuronas de áreas corticales transmodales interconectadas entre sí, que son coactivadas con el propósito de mediar rendimientos cognitivos especializados. La formalización de estos sistemas anatómicamente distintos fue descrita por Mesulam 6 , quien propuso 5 redes:

1. Red atencional espacial cuyas áreas claves son la corteza parietal posterior y los campos frontales oculares.

2. Red de lenguaje anclada en las áreas de Broca y Wernicke en el hemisferio izquierdo.

3. Red de memoria explícita anclada en el complejo hipocámpico-corteza entorrinal y corteza parietal inferior.

4. Red de reconocimiento de fisonomías y objetos anclado en la corteza inferotemporal.

5. Red ejecutiva de control frontoparietal que está compuesta por la corteza prefrontal rostro lateral, ínsula anterior, corteza cingulada anterior y lóbulo parietal inferior.

Más recientemente se agregó una interesante red denominada red neural por defecto (RND) ${ }^{7}$. Esta red se activa cuando el individuo está despierto en reposo, y se inactiva durante la realización de tareas que demandan atención. La RND permanece activa y sincronizada cuando el individuo está centrado en el mundo interior y también durante la anestesia y fases iniciales del sueño ${ }^{8}$. Esta nueva red ha sido involucrada en varias enfermedades cerebrales como enfermedad de Alzheimer, el autismo, la esquizofrenia, la depresión y el déficit atencional ${ }^{9}$. En esta revisión nos abocaremos a describir la red por defecto y su rol en la enfermedad de Alzheimer.

\section{Red neural por modo de defecto}

La "red neural por defecto" ("default mode network", DMN por sus siglas en inglés), fue acuñada por Marcus Raichle y cols. Es una red funcional prominente, caracterizada por una importante actividad intrínseca de un set de regiones cerebrales que están activas cuando el individuo mantiene actividad de vagabundeo mental, y disminuyen su actividad cuando el individuo ejerce actividades perceptivas o motoras ${ }^{7}$. Al preguntarles a los participantes qué pensaban en los momentos de reposo se determinó que correspondía a una mezcla de recuerdos, planes futuros, pensamientos y experiencias personales, es decir, actividad introspectiva o autoreferencial.

\section{Anatomía de la red neural por defecto}

Originalmente se estableció la anatomía de la RND por los aumentos de activación durante tareas pasivas comparadas con un amplio rango de tareas activas ${ }^{10}$. Aproximaciones más recientes han identificado este sistema vía correlación de actividad intrínseca en distintas áreas cerebrales ${ }^{11,12}$. Las áreas corticales implicadas en la red por defecto incluyen áreas de asociación y no involucran áreas motoras o sensoriales. Incluyen la corteza prefrontal medial (MPFC), corteza cingulada anterior, precuneus, cíngulo posterior, corteza parietal lateral (giro supramarginal y angular) y lóbulo temporal lateral. Las regiones dentro de esta red convergen en la corteza cingulada posterior extendiéndose al precuneus que está conectado fuertemente con el hipocampo.

\section{Relación entre metabolismo y actividad funcional cerebral}

La actividad cerebral relacionada a tareas habitualmente se mide por el aumento del flujo sanguíneo cerebral mediante técnicas de tomografía por emisión de positrones (PET) y resonancia 
magnética funcional (fMRI) ${ }^{13,14}$. La actividad basal de reposo se sustrae lográndose así aislar el componente activo relacionado a la tarea. Raichle y cols observaron que la actividad cerebral de reposo que se restaba en estos estudios era notoriamente alta y decidieron estudiarla per se. Las primeras evidencias de esta actividad cerebral de reposo sin esfuerzo perceptivo ni cognitivo fueron sospechadas del trabajo de Sokoloff y cols ${ }^{15}$, quienes encontraron que no había grandes cambios del metabolismo cerebral ante una demanda cognitiva, lo que implicaba que el cerebro en reposo tiene una importante actividad metabólica, que se incrementa ligeramente con la realización de una tarea.

La evidencia definitiva de la existencia de esta red neural proviene de la medición de cambios de flujo sanguíneo cerebral por PET y detección de cambios en la concentración de oxígeno en la sangre por fMRI (BOLD, blood oxygen level dependent), que reflejan flujo sanguíneo ${ }^{7}$. En la ausencia de aferencias perceptivas y manifestaciones conductuales se aprecian fluctuaciones espontáneas de la señal BOLD generadas por fluctuaciones espontáneas de la actividad neuronal, que cumplen los criterios de una actividad neuronal intrínseca del cerebro, hipótesis que se confirma al comparar las señales con la actividad eléctrica cerebral ${ }^{10}$.

La conectividad de la RND se puede medir analizando la conectividad funcional mediante fMRI (fc-fNRI, functional connectivity fMRI) en reposo. Esta técnica estima la coherencia de la actividad neural en distintas regiones cerebrales midiendo los patrones de fluctuaciones sincrónicas de la señal de BOLD fMRI. Esta es una técnica promisoria para medir integridad de redes neurales ya que no es invasiva, no implica inyección de medio de contraste ni radiactivo, y al ser una medición en reposo permite el estudio de la integridad funcional en personas incapaces de comprender las órdenes de una tarea, como aquellas con demencias avanzadas $u$ otras enfermedades siquiátricas.

Una manera de evaluar la importancia relativa de la actividad cerebral intrínseca en comparación con la evocada es examinando el costo del consumo de energía. El cerebro -que representa 2\% del peso corporal- consume $20 \%$ de la energía del cuerpo humano. Los cambios ante la actividad evocada por una tarea específica son muy pequeños respecto de la actividad basal $(<5 \%)$, de lo que se deduce que la mayor parte de la energía consumida por el cerebro está dedicada a la actividad intrínseca ${ }^{14}$, que en su mayor parte, 60-80\% está dedicada al reciclaje de glutamato, esto es, señales neurales.

La energía cerebral se obtiene de la glicólisis y la fosforilación oxidativa. La fosforilación oxidativa en las mitocondrias produce 30 moléculas de ATP por molécula de glucosa, en tanto que la glicólisis produce sólo 2, de manera que la fosforilación oxidativa es la mayor fuente de energía para la actividad cerebral. Sin embargo, la glicolisis presenta ventajas ya que es capaz de producir ATP en forma más rápida que la fosforilación oxidativa.

\section{La glicólisis aeróbica y red neural por defecto}

La glicólisis aeróbica, conocida como el fenómeno de Warburg, por su descubridor, fue descrita originalmente en el metabolismo de tumores ${ }^{16}$ y se caracteriza por el metabolismo de glucosa a lactato en presencia de suficiente oxígeno, lo que en células diferenciadas normalmente sólo ocurre en ausencia de oxígeno. De ahí su nombre de glicólisis aeróbica.

Las neuronas, al igual que las células neoplásicas, utilizan glucosa en forma importante a través de lo que se denomina glicólisis aeróbica. La glicólisis aeróbica es sumamente eficiente cuando se requiere cambios rápidos en la actividad cerebral, sin embargo, ha recibido poca atención porque la mayor parte de la investigación se ha centrado en el rol de la glucosa en la fosforilación oxidativa ${ }^{17}$.

La glicólisis aeróbica da cuenta de 10-20\% de la glucosa consumida por el cerebro adulto ${ }^{17}$. De este porcentaje, en condiciones de reposo, los astrocitos aportan $85 \%$ y este alto nivel se atribuye al reciclaje de glutamato. Las neuronas contribuyen en forma a mínima al consumo de glucosa. Además, neuronas y astrocitos exhiben diferentes formas de utilización de la glucosa. Los astrocitos consumen glucosa preferentemente través de glicólisis a piruvato y lactato, en cambio las neuronas lo hacen más bien a través del ciclo de las pentosas ${ }^{18,19}$.

La glicolisis aeróbica cumple varias funciones. La rápida producción de ATP de la glicólisis aeróbica la hace muy efectiva para acomodarse a cambios rápidos de requerimiento de energía por la Na/K/ATPasa, necesaria para la extrusión 
de sodio después de la depolarización y para la remoción de glutamato de las sinapsis por parte de los astrocitos. Otra función de la glicólisis aeróbica es la producción de sustratos para la biosíntesis de nucleótidos, ácidos grasos y aminoácidos a través de la vía de las pentosas. Es usada como materia prima para la síntesis de macromoléculas y neurotransmisores, necesarios para la actividad sináptica, y no como fuente de energía. Finalmente, la glicólisis aeróbica es crítica para la sobrevida de las neuronas a través de la regulación de la apoptosis. Esto lo hace a través de su efecto en el estado de óxido-reducción cerebral (relación NAD /NADH) produciendo NADH por el ciclo de las pentosas, que es crítico en la reducción de moléculas de oxígeno reactivas y control de la apoptosis.

Vaishnavi y cols ${ }^{17}$ describieron recientemente que la glicólisis aeróbica es mayor en dos sistemas corticales funcionales. Calcularon los niveles de glicólisis aeróbica, midiendo la tasa metabólica de $\mathrm{O}_{2}$, la tasa metabólica de glucosa y el flujo cerebral mediante PET y encontraron que la glicólisis aeróbica es mayor al promedio en la RND y en áreas frontales dorsolaterales y parietales relacionadas con procesos de control de tareas. Al revés, encontraron que los niveles de glicólisis aeróbica eran menores que el promedio en el cerebelo y en temporal inferior, incluyendo el hipocampo.

\section{Enfermedad de Alzheimer (EA)}

El mecanismo patogénico primario en la EA es aún desconocido. La hipótesis más aceptada es la hipótesis del amiloide que propone que el evento inicial es una acumulación excesiva del péptido beta amiloide $(A \beta)$, que se agrega para formar las placas amiloideas, provocando fosforilación y agregación de tau en ovillos neurofibrilares, inflamación, degeneración de sinapsis y finalmente muerte neuronal y demencia ${ }^{20}$. El amiloide se deposita precozmente en ciertas regiones, como la corteza cingulada posterior y el precuneo, zonas pertenecientes a la RND, y no está claro el mecanismo que explica esta vulnerabilidad regional selectiva ${ }^{21}$.

La evidencia más precoz de que la RND está alterada en la EA proviene de estudios de metabolismo de glucosa en reposo. El patrón de hipometabolismo incluye las regiones posteriores de la RND incluyendo la corteza cingulada poste- rior y precuneus, corteza parietal lateral inferior y temporal lateral ${ }^{21}$ y en las etapas más avanzadas lóbulos frontales y todo el cerebro. Grecius y cols ${ }^{22}$ demostraron que la conectividad del hipocampo con las otras regiones de la RND, tales como corteza prefrontal medial y corteza cingulada posterior, estaban reducidas en pacientes con EA en comparación con sujetos sanos.

Interesantemente, estudios in vivo en los cuales se visualiza el depósito de $A \beta$ en conjunto con fMRI demostró que hay coincidencia entre las regiones de mayor depósito de $\mathrm{A} \beta$ y la $\mathrm{RND}^{21}$. La RND es especialmente vulnerable a alteraciones metabólicas, atrofia y depósito de A $\beta$. Pacientes con EA y deterioro cognitivo leve (DCL) muestran una disrupción selectiva de la RND, especialmente en la conectividad entre el precúneo y cingulado posterior con el hipocampo y corteza prefrontal medial $^{22,23}$. Además, la menor conectividad de la RND permitió predecir aquellos pacientes con DCL que progresarían a $\mathrm{EA}^{24}$. Incluso en individuos cognitivamente normales, aquellos con depósito de $A \beta$ tienen menor conectividad de la RND en regiones similares a los pacientes con EA y DCL ${ }^{25}$. La menor conectividad de la RND en reposo se asocia además a una falla en la deactivacion inducida por la realización de tareas ${ }^{26}$. Por lo tanto, la RND en estos pacientes presenta una menor conectividad en reposo y una menor deactivación durante una tarea.

En estudios recientes Bero y cols $^{27}$ sugieren que los niveles intersticiales de $\mathrm{A} \beta$ están controlados por la actividad sináptica local. Mediante microdiálisis in vivo del espacio intersticial de cerebro de ratón modelo de Alzheimer, demostraron que los niveles de lactato, producto del metabolismo neuronal y marcador de la glicolisis aeróbica, aumenta con el aumento de la actividad neuronal y se correlaciona con la concentración de $A \beta$ soluble. Cuando la actividad neuronal aumenta la concentración de $\mathrm{A} \beta$ soluble aumenta y las placas de amiloide aumentan en número y tamaño, y a la inversa cuando la actividad neuronal se reduce los niveles de $A \beta$ disminuyen y no se desarrollan las placas seniles ${ }^{27}$.

En las etapas precoces de la EA la utilización cerebral de glucosa se reduce en hasta $45 \%$ y el flujo sanguíneo cerebral $\sim 18 \%$; en etapas más avanzadas se aprecian reducciones de $55-65 \%$ del flujo sanguíneo cerebra ${ }^{28}$. Estos defectos son más prominentes en la RND, que normalmente se 
caracteriza por una alta actividad metabólica y glicólisis aeróbica. El consumo de energía neuronal se correlaciona con el reciclaje de neurotransmisores de modo que aquellas regiones más activas, como la RND, son más susceptibles de depósito de amiloide. El depósito de amiloide a su vez se asocia a menor deactivación de la RND que se mantiene activa durante tareas. Así, una elevación crónica de la actividad neuronal endógena sería un factor predisponente de depósito de amiloide ya que la estimulación neuronal determina mayor liberación de amiloide al espacio intersticial.

Recientemente se ha descrito que variaciones genéticas en la gliceraldehido-3-fosfato deshidrogenasa, enzima clave en la glicólisis para la generación de ATP y necesaria para la actividad de la NA,K ATPasa en astrocitos y densidades postsinapticas, es factor predisponente de Alzheimer, lo que apoya una conexión entre la glicólisis y la neurodegeneración ${ }^{29}$. Una falla en la capacidad de mantener una adecuada glicólisis aeróbica, necesaria para actividad sináptica, manejo de stress oxidativo y biosíntesis de proteínas, neurotrasmisores, lípidos y ácidos nucleicos jugaría un rol en la fisiopatología de la EA.

El estudio de las alteraciones en el funcionamientos de la RND y metabolismo de glicólisis aeróbica y su relación con la enfermedad de Alzheimer abren nuevas vías de investigación que podrían desembocar en nuevas estrategias de tratamiento, tan esperadas para esta devastadora enfermedad del siglo XXI.

\section{Referencias}

1. Hartline HK. The response of single optic nerve fibers of the vertebrate eye to illumination of the retina. Am J Physiol 1938; (121): 400-15.

2. Brown $\mathrm{G}$. The intrinsic factors in the act of progression in the mammal. Proc Natl Acad Sci U S A 1914; 84: 30819.

3. Yuste R, MacLean JN, Smith J, Lansner A. The cortex as a central pattern generator. Nat Rev Neurosci 2005; 6 (6): 477-83.

4. Llinás R, Ribary U, Contreras D, Pedroarena C. The neuronal basis for consciousness. Philos Trans R Soc Lond B Biol Sci 1998; 353 (1377): 1841-9.

5. Dehaene S, Brannon EM. Space, time, and number: a Kantian research program. Trends Cogn Sci 2010; 14 (12): 517-9.
6. Mesulam MM. Spatial attention and neglect: parietal, frontal and cingulate contributions to the mental representation and attentional targeting of salient extrapersonal events. Philos Trans R Soc Lond B Biol Sci 1999; 354 (1387): 1325-46.

7. Raichle ME, MacLeod AM, Snyder AZ, Powers WJ, Gusnard DA, Shulman GL. A default mode of brain function. Proc Natl Acad Sci U S A 2001; 98 (2): 676-82.

8. Greicius MD, Kiviniemi V, Tervonen O, Vainionpaa V, Alahuhta S, Reiss AL, et al. Persistent default-mode network connectivity during light sedation. Hum Brain Mapp 2008; 29 (7): 839-47.

9. Zhang D, Raichle ME. Disease and the brain's dark energy. Nat Rev Neurol 2010; 6 (1): 15-28.

10. He BJ, Snyder AZ, Zempel JM, Smyth MD, Raichle ME. Electrophysiological correlates of the brain's intrinsic large-scale functional architecture. Proc Natl Acad Sci USA 2008; 105 (41): 16039-44.

11. Buckner RL, Andrews-Hanna JR, Schacter DL. The brain's default network: anatomy, function, and relevance to disease. Ann N Y Acad Sci 2008; 1124: 1-38.

12. Greicius MD, Supekar K, Menon V DRF. Resting-state functional connectivity reflects structural connectivity in the default mode network. Proc Natl Acad Sci 2012; 19: $72-8$

13. Lassen NA, Ingvar DH, Skinhoj E. Brain function and blood flow. Sci Am 1978; 239 (4): 62-71.

14. Burton H, MacLeod AM, Videen TO, Raichle ME. Multiple foci in parietal and frontal cortex activated by rubbing embossed grating patterns across fingerpads: a positron emission tomography study in humans. Cereb Cortex 1997; 7 (1): 3-17.

15. Sokoloff L, Mangold R, Wechsler RL, Kenney C, Kety SS. The effect of mental arithmetic on cerebral circulation and metabolism. J Clin Invest 1955; 34 (7, Part 1): 1101-8.

16. Warburg O. [Origin of cancer cells]. Oncología 1956; 9 (2): 75-83.

17. Vaishnavi SN, Vlassenko AG, Rundle MM, Snyder AZ, Mintun MA, Raichle ME. Regional aerobic glycolysis in the human brain. Proc Natl Acad Sci USA 2010; 107 (41): 17757-62.

18. Bolanos JP, Almeida A. The pentose-phosphate pathway in neuronal survival against nitrosative stress. IUBMB Life 2010; 62 (1): 14-8.

19. Bolanos JP, Almeida A, Moncada S. Glycolysis: a bioenergetic or a survival pathway? Trends Biochem Sci 2010; 35 (3): 145-9.

20. Hardy J, Selkoe DJ. The amyloid hypothesis of Alzheimer's disease: progress and problems on the road to therapeutics. Science 2002; 297 (5580): 353-6. 
21. Buckner RL, Snyder AZ, Shannon BJ, LaRossa G, Sachs R, Fotenos AF, et al. Molecular, structural, and functional characterization of Alzheimer's disease: evidence for a relationship between default activity, amyloid, and memory. J Neurosci 2005; 25 (34): 7709-17.

22. Greicius MD, Menon V. Default-mode activity during a passive sensory task: uncoupled from deactivation but impacting activation. J Cogn Neurosci 2004; 16 (9): 1484-92.

23. Wu X, Li R, Fleisher AS, Reiman EM, Guan X, Zhang $\mathrm{Y}$, et al. Altered default mode network connectivity in Alzheimer's disease--a resting functional MRI and Bayesian network study. Hum Brain Mapp 2011; 32 (11): 1868-81.

24. Petrella JR, Sheldon FC, Prince SE, Calhoun VD, Doraiswamy PM. Default mode network connectivity in stable vs progressive mild cognitive impairment. Neurology 2011; 76 (6): 511-7.

25. Hedden T, Van Dijk KR, Becker JA, Mehta A, Sperling RA, Johnson KA, et al. Disruption of functional con- nectivity in clinically normal older adults harboring amyloid burden. J Neurosci 2009; 29 (40): 12686-94.

26. Pihlajamäki M, O' Keefe K, Bertram L, Tanzi RE, Dickerson BC, Blacker D, et al. Evidence of altered posteromedial cortical FMRI activity in subjects at risk for Alzheimer disease. Alzheimer Dis Assoc Disord 2010; 24 (1): 28-36.

27. Bero AW, Yan P, Roh JH, Cirrito JR, Stewart FR, Raichle ME, et al. Neuronal activity regulates the regional vulnerability to amyloid-beta deposition. Nat Neurosci 2011; 14 (6): 750-6.

28. Hoyer S, Nitsch R. Cerebral excess release of neurotransmitter amino acids subsequent to reduced cerebral glucose metabolism in early-onset dementia of Alzheimer type. J Neural Transm 1989; 75 (3): 227-32.

29. Li Y, Nowotny P, Holmans P, Smemo S, Kauwe JS, Hinrichs AL, et al. Association of late-onset Alzheimer's disease with genetic variation in multiple members of the GAPD gene family. Proc Natl Acad Sci USA 2004; 101 (44): 15688-93. 